\title{
CpG single nucleotide polymorphisms of the insulin signaling pathway associated with the risk of prediabetic status/type 2 diabetes in Chinese population
}

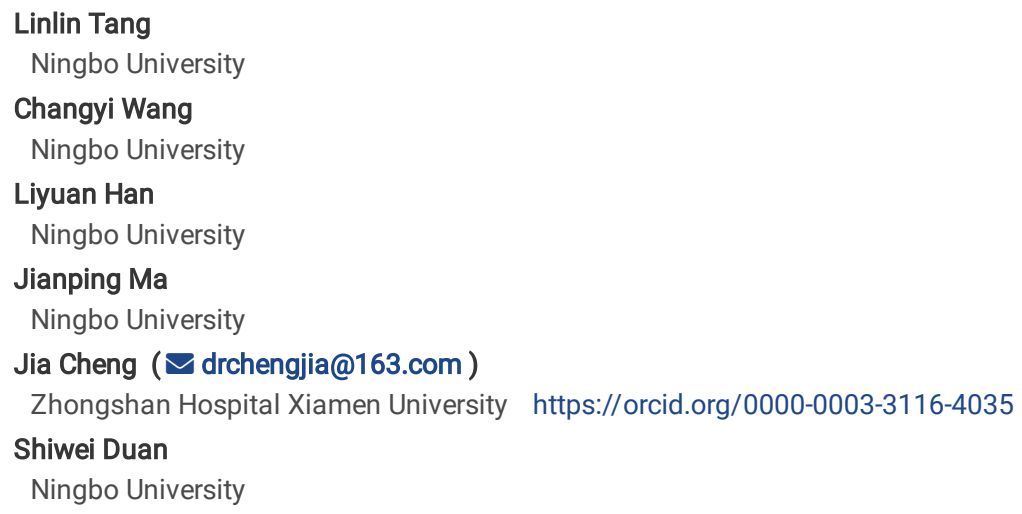




\section{Abstract}

Aims: Cytosine-phosphate-guanine single nucleotide polymorphisms (CpG SNPs) have been identified as new markers in human diseases. We aimed to evaluate the contribution of 29 CpG SNPs in the insulin signaling pathways to the risk of prediabetes and type 2 diabetes (T2D) in Han Chinese.

Materials and methods: We recruited 708 T2D subjects, 253 prediabetes subjects and 575 healthy controls from 16 community health service centers (CHSC) in Shenzhen Nanshan district. Detailed clinical information and DNA samples were collected. A total of 29 CpG SNPs of the insulin signaling pathways were sequenced using the MassARRAY platform. Binary logistic regression was applied to evaluate the association between these 29 CpG SNPs with prediabetes and T2D after adjusted the relevant confounding factors. The potential interactions among the $29 \mathrm{CpG}$ SNPs were conducted by the generalized multifactor dimensionality reduction (GMDR).

Results: Significant associations are found between KRAS rs7311692 [Padjusted $=0.009,0 R=0.562(0.363-0.868)$ by allele] and CBLC rs2965143 [Padjusted $=0.008, \mathrm{OR}=1.591(1.128-2.242)$ under the dominant model] with prediabetes. A further breakdown analysis by gender identify a significant association between MAPK9 rs1363513 and T2D risk in males [Padjusted $=0.004,0 R=0.57(0.399-0.836)$ by allele], while FLOT2 rs4795473 is associated with prediabetes in females $(P=0.007$ by genotype). However, no significant effect of potential interaction is observed among the 29 CpG SNPs.

Conclusion: These results indicated that KRAS rs7311692, CBLC rs2965143 and FLOT2 rs4795473 are related with prediabetes, while MAPK9 rs1363513 was associated with male T2D.

\section{Introduction}

Type 2 diabetes (T2D) is a complex human disease contributed by genetic, environmental factors, and their interactions[1]. The incidence of T2D is increasing rapidly in both developed and developing countries[1]. As an intermediate process between normal glucose tolerance (NGT) and T2D, prediabetes is the major risk factor of T2D and its incidence is also soaring in recent years[1].

Single nucleotide polymorphisms (SNPs) have been identified as genetic markers of T2D. A large number of susceptibility loci have been validated in different ethnic populations according to the Genome Wide Association Studies (GWAS)[2]. However, all of the known genetic polymorphisms together can only account for about $5 \%$ of the phenotypic variance, therefore there is considerable missing heritability of T2D.

Recently, epigenetic mechanisms including DNA methylation and histone modification et al are found to be involved in the regulation of $\beta$-cell function in pancreatic islets[3]. DNA methylation is regarded as a bridge that connected the environmental influence and genetic background, that plays an important role in the pathogenesis of T2D. Interestingly, DNA methylation usually occurs on the $\mathrm{CpG}$ sites, which may influence the gene expression by regulating the transcription process [4]. The DNA methylation levels are commonly analyzed at clusters of CpG sites in the gene promoter and are used for indication of epigenetic effects[5]. It has been assumed that the gene functions may be influenced by disease related SNPs that correlating with DNA methylation status, and the introduction or removal of the CpG sites may contribute to the molecular mechanism of T2D development[3]. Moreover, CpG-SNPs have been found to be associated with many human diseases, such as coronary heart disease, schizophrenia, obesity et al[6, 7]. Human CpG SNPs might act as new potential molecular markers in many diseases.

In this study, we hypothesize that CpG SNPs (SNPs involved in the CpG sites) of the insulin signal pathway may be new markers for the development of prediabetes and T2D, and there may be potential interactions among the $29 \mathrm{CpG}$ SNPs in insulin signal pathway. Many SNPs of the candidate genes in insulin signal pathway have been identified as significant loci for T2D. Furthermore, it has been reported that CpG SNPs contributed to T2D onset by changing the DNA methylation levels in human pancreatic islets. Thereby, the purpose of our study is to explore the association between CpG SNPs in insulin signal pathway and the susceptibility of prediabetes and T2D, which is helpful to elucidated their clinical value in T2D development.

\section{Materials And Methods}

\subsection{Samples collection}

A total of 1,536 subjects consisted of 708 T2D subjects, 253 prediabetes subjects and 575 healthy controls were included in our study. The subjects were collected from 16 community health service centers (CHSC) in Shenzhen Nanshan district of China. The subiects of T2D and prediabetes were diagnosed according to the American Diabetes Association guidelines. Subjects with hypertension, coronary heart disease or other serious diseases were excluded in this study. Detailed information about age, body mass index (BMI), waist: hip ratio (WHR), systolic blood pressure (SBP), diastolic blood pressure (DBP), total cholesterol (TC), triglycerides (TG), low density lipoproteins (LDL), uric acid (UA), creatinine (Cre) were also documented. The study protocol was approved by the Ethical Committee of Shenzhen Nanshan Center for Chronic Disease Control, and the informed written consents were obtained from all the subjects.

\subsection{CpG-SNP selection}

The selection criteria of CpG-SNPs were as follows: (1) the selected CpG-SNPs were on the promoter of genes in insulin signal pathway; (2) the minor frequencies of the selected CpG-SNPs were over 10\% in HapMap HCB population; (3) CpG-SNPs with design problems or failed assays were excluded. Finally, 29 CpG-SNPs in insulin signal pathway were included in our study (Figure 1). All of the CpG-SNPs were able to introduce or remove a CpG site.

SNP genotyping

Genomic DNA was extracted from peripheral blood lymphocytes using the nucleic acid extraction analyzer (Lab-Aid 820, Xiamen, China). DNA concentration was determined using the NanoDrop 1000 spectrophotometer (Thermal Scientific Co. Ltd., Wilmington, USA). The PCR cycles included an initial denaturation 
stage $94^{\circ} \mathrm{C}$ for $15 \mathrm{sec}, 45$ cycles of denaturation for $20 \mathrm{sec}$ at $94^{\circ} \mathrm{C}$, an annealing phase conducted at $56^{\circ} \mathrm{C}$ for 30 sec, a primer extension at $72{ }^{\circ} \mathrm{C}$ for 1 min and a final extension for 3 minutes at $72^{\circ} \mathrm{C}$. Genotyping of the $29 \mathrm{CpG}$-SNPs was performed on the Sequenom MassARRAY platform. The primer sequences of the 29 CpG-SNPs were shown in Table 1.

\subsection{Statistical analysis}

Continuous variables among the three groups were analyzed by one-way analysis of variance. The genotype and allele frequencies of CpG-SNPs between cases and controls were analyzed by Chi-square test under dominant and recessive models. Binary logistic regression analysis was used to analyze the association between CpG-SNPs and prediabetes/T2D risk after adjusted the corresponding confounders. Besides, we also conducted the sub-group analysis stratified by genders.

To reduce type I error induced by multiple tests, $\mathrm{P}<0.01$ was adopted as the significant threshold. The above statistical tests were performed by SPSS software (SPSS Inc., version 16.0, Chicago, IL, USA). The consistency of genotype frequencies (Hardy-Weinberg equilibrium, HWE) were performed by Arlequin program (version 3.5). The potential interactions among the 29 CpG-SNPs were performed by generalized multifactor dimensionality reduction (GMDR). A twosided $\mathrm{P}<0.05$ were considered to be significant.

\section{Results}

The characteristics of the included 1536 subjects were presented in Supplemental Table 1. There were significant differences of clinical features among the three groups in different comparisons. The comparison results between insulin signal pathway genotype distributions and allele frequencies among T2D subjects, prediabetes subjects and healthy controls were summarized in Table 2.

Significant association between KRAS rs7311692 and prediabetes was revealed in the multivariate analysis after adjusted for age, BMI, SBP, DBP, TC, TG, UA and Cre [prediabetes/controls: $P_{\text {adjusted }}=0.009, \mathrm{OR}=0.562(0.363-0.868)$ by allele]. In addition, we found a significant association between $C B L C$ rs2965143 and prediabetes following the adjustment of age, BMI, SBP, DBP, TC, TG, UA, Cre [prediabetes/controls: $\mathrm{P}_{\text {adjusted }}=0.008, \mathrm{OR}=1.591(1.128-2.242)$ under the dominant model].

A further breakdown analysis by gender revealed that MAPK9 rs1363513 was significantly associated with T2D risk following the adjustment of age, BMI, WHR, SBP, DBP, TG, IDL,UA and Cre in male subjects [T2D/controls: $P_{\text {adjusted }}=0.004, O R=0.57(0.399-0.836)$ by allele] as shown in Supplemental Table 2. We also observed a significant association between FLOT2 rs4795473 and prediabetic status in females (prediabetes/controls: $\mathrm{P}$ adjusted $=0.007$ by genotype). However, we didn't observe any potential interaction among these 29 CpG-SNPs by GMDR analysis (data not shown).

\section{Discussion}

In this study, we investigated the association between T2D risk and 29 CpG SNPs of gene promoters that selected from insulin signal pathway in 1536 subjects. Our study revealed that the allele frequencies of KRAS rs7311692 and CBLC rs2965143 were associated with prediabetic status. As the influence of gender can contribute to the development of T2D, which is mainly due to the mediation of sex hormones, we also performed a subgroup analysis stratified by gender. Significant associations of MAPK9 rs1363513 with male T2D and FLOT2 rs4795473 with female prediabetes were observed.

To the best of our knowledge, this is the first report about CpG SNPs with susceptibility to status of prediabetes and T2D. Prediabetes is an intermediate stage of hyperglycemia, which is a high risk factor of T2D onset [8]. This study reveal that KRAS rs7311692, CBLC rs2965143 and FLOT2 rs4795473 were associated with prediabetes. It has been reported that there was an interaction between glucose levels and diabetes risk SNPs, which perform an effect on the insulin secretion[9-11]. Therefore, the T2D/prediabetes related CpG SNPs might have similar biological effect on insulin secretion that resulting in blood glucose variability. Additionally, prediabetes related SNPs, such as at PERK rs6750998 and XBP1 rs2239815, were also found associated with homeostasis model assessments of insulin resistance[12]. Interestingly, we observe significant associations for KRAS rs7311692, CBLC rs2965143 and FLOT2 rs4795473 with prediabetic status. Our study provide new genetic evidences indentified contributions of CpG SNPs of insulin signal pathway to the molecular pathologic mechanism of insulin resistance in human prediabetes.

Insulin resistance and $\beta$-cell dysfunction are main crucial metabolic disorder in T2D[13], a large number of SNPs of T2D candidate genes exerted an influence on the onset of T2D by regulating the insulin secretion and sensitivity. Taqi et al reported that T2D-associated SNPs could affect gene function by altering its DNA methylation level[14]. Similar mechanism existed between the CpG SNPs and DNA methylation status, that can influence the binding ability of DNAbinding proteins resulted in gene regulation. It is important to understand the molecular mechanisms of CpG SNPs of insulin signal pathway for further clarifying the pathogenesis of T2D.

DNA methylation is critical for gene transcription regulation and chromosome conformation[15]. In general case, increased DNA methylation level of the promoter results in decreased gene expression. In order to understand the mechanism of T2D, it is necessary to validate the link between CpG SNPs and DNA methylation. Hence, the analyses of DNA methylation status that surrounding the CpG SNP sites of the insulin signal pathway are needed for further epigenetic study.

The merits of our study include: firstly, we recruited a homogeneous population with strict inclusion and exclusion criteria; secondly, our study may provide a novel hint in clarifying the molecular mechanism of prediabetes; thirdly, we explored the potential interactions among the 29 CpG SNPs with GMDR method. However, there are some limitations in our study: the sample size is not very large and we didn't test the relationship between the prediabetes/T2D related CpG SNPs and DNA methylation levels. 


\section{Conclusions}

In conclusion, we found four CpG SNPs are significantly associated with prediabetes/T2D in the insulin signal pathways. These genetic locus may be potential biomarkers with clinical value in the assessment of the T2D risk.

\section{Declarations}

\section{Funding}

The research was supported by grants from Shenzhen Nanshan Bureau of Science and Technology (2010058), K.C.Wong Magna Fund in Ningbo University, the National Natural Science Foundation of China $(31100919$, 81371469 and 81402745), the Natural Science Foundation of Ningbo (2014A610235 and 2014A610268), the Natural Science Foundation of Zhejiang Province (LR13H020003 and LQ13H260002), Zhejiang Provincial Bureau of Traditional Chinese Medicine (2013ZZ003) and the Sciences Technology Department of Zhejiang Province (2013F2005), Zhejiang Province Scientific Research Projects of Education (Y201326971), the Ministry of Education, Humanities and Social Sciences project (14YJC630046), Ningbo University Scientific Research Fund (XKL14D2098) and open fund of Zhejiang Provincial Key Laboratory of Pathophysiology (201503).

\section{Author's contributions}

Changyi Wang and Shiwei Duan contributed to the design of the study. Liyuan Han performed statistical analysis; Jia Cheng and Linlin Tang contributed to the drafting of the manuscript; all authors participated in generation, collection, assembly, analysis, and interpretation of data. All authors read and approved the final manuscript.

\section{Declaration of Competing Interests}

The authors declare that they have no conflict of interest.

\section{References}

1. Ginter E, Simko V: Type 2 diabetes mellitus, pandemic in 21st century. Advances in experimental medicine and biology 2012, 771:42-50.

2. Saxena R, Elbers CC, Guo Y, Peter I, Gaunt TR, Mega JL, Lanktree MB, Tare A, Castillo BA, Li YR et al: Large-scale gene-centric meta-analysis across 39 studies identifies type 2 diabetes loci. American journal of human genetics 2012, 90(3):410-425.

3. Dayeh TA, Olsson AH, Volkov P, Almgren P, Ronn T, Ling C: Identification of CpG-SNPs associated with type 2 diabetes and differential DNA methylation in human pancreatic islets. Diabetologia 2013, 56(5):1036-1046.

4. Bird A: Perceptions of epigenetics. Nature 2007, 447(7143):396-398.

5. Gilbert ER, Liu D: Epigenetics: the missing link to understanding beta-cell dysfunction in the pathogenesis of type 2 diabetes. Epigenetics 2012, 7(8):841852.

6. Mansego ML, Milagro FI, Zulet MA, Martinez JA: SH2B1 CpG-SNP is associated with body weight reduction in obese subjects following a dietary restriction program. Annals of nutrition \& metabolism 2015, 66(1):1-9.

7. Shen L, Kondo Y, Guo Y, Zhang J, Zhang L, Ahmed S, Shu J, Chen X, Waterland RA, Issa JP: Genome-wide profiling of DNA methylation reveals a class of normally methylated CpG island promoters. PLoS genetics 2007, 3(10):2023-2036.

8. Bansal N: Prediabetes diagnosis and treatment: A review. World journal of diabetes 2015, 6(2):296-303.

9. Kempf K, Rathmann W, Herder C: Impaired glucose regulation and type 2 diabetes in children and adolescents. Diabetes/metabolism research and reviews 2008, 24(6):427-437.

10. Eades CE, Leese GP, Evans JM: Incidence of impaired glucose regulation and progression to type 2 diabetes mellitus in the Tayside region of Scotland Diabetes research and clinical practice 2014, 104(1):e16-19.

11. Heni M, Ketterer C, Thamer C, Herzberg-Schafer SA, Guthoff M, Stefan N, Machicao F, Staiger H, Fritsche A, Haring HU: Glycemia determines the effect of type 2 diabetes risk genes on insulin secretion. Diabetes 2010, 59(12):3247-3252.

12. Feng N, Ma X, Wei X, Zhang J, Dong A, Jin M, Zhang H, Guo X: Common variants in PERK, JNK, BIP and XBP1 genes are associated with the risk of prediabetes or diabetes-related phenotypes in a Chinese population. Chinese medical journal 2014, 127(13):2438-2444.

13. Yabe D, Seino Y, Fukushima M, Seino S: beta cell dysfunction versus insulin resistance in the pathogenesis of type 2 diabetes in East Asians. Current diabetes reports 2015, 15(6):602.

14. Taqi MM, Bazov I, Watanabe H, Sheedy D, Harper C, Alkass K, Druid H, Wentzel P, Nyberg F, Yakovleva T et al: Prodynorphin CpG-SNPs associated with alcohol dependence: elevated methylation in the brain of human alcoholics. Addiction biology 2011, 16(3):499-509.

15. Dastani Z, Hivert MF, Timpson N, Perry JR, Yuan X, Scott RA, Henneman P, Heid IM, Kizer JR, Lyytikainen LP et al: Novel loci for adiponectin levels and their influence on type 2 diabetes and metabolic traits: a multi-ethnic meta-analysis of 45,891 individuals. PLoS genetics 2012, 8(3):e1002607.

\section{Tables}


Table 1. Primer sequences and the information of 29 CpG-SNPs

\begin{tabular}{|c|c|c|c|c|c|}
\hline Gene & $\mathrm{Chr}$ & SNP & Primer & Sequence $\left(5^{\prime}-3^{\prime}\right)$ & Extension primer sequence $\left(5^{\prime}-3^{\prime}\right)$ \\
\hline \multirow[t]{2}{*}{$P I K 3 C D$} & 1 & rs7518602 & 1st-primer & ACGTTGGATGATATTTTGGGGTGGCACACG & gcatGACACACACCCAAAATGTGACT \\
\hline & & & 2nd-primer & ACGTTGGATGTTGCGAGGTGACCAGTGTTG & \\
\hline \multirow[t]{2}{*}{$M K N K 1$} & 1 & rs3753359 & 1st-primer & ACGTTGGATGCAGAGGCTGCAGTGTTTATG & GATGCCGCATCACCCT \\
\hline & & & 2nd-primer & ACGTTGGATGCAGAGCATCCACCTATTGAG & \\
\hline \multirow[t]{2}{*}{$P P P 1 C B$} & 2 & rs7598876 & 1st-primer & ACGTTGGATGCTCTCTCCACGTCCACGGC & ggggaGGCGCCTGCGCCACCG \\
\hline & & & 2 nd-primer & ACGTTGGATGTCTGCGTAACCGGGACTTC & \\
\hline \multirow[t]{2}{*}{$P R K C I$} & 3 & rs481781 & 1st-primer & ACGTTGGATGGCTGATCATGGCATCAAAC & ctcg CGCGTGAAAGCACCCCTC \\
\hline & & & 2nd-primer & ACGTTGGATGCTAGAGCTGGTCATTGTCTC & \\
\hline \multirow[t]{2}{*}{ MAPK9 } & 5 & rs1363513 & 1st-primer & ACGTTGGATGATGGGGAAAACCGTGGAGAG & CCACTCTTCTAGTCCСCAA \\
\hline & & & 2nd-primer & ACGTTGGATGGTCAACCATTGTTCCCACTC & \\
\hline \multirow[t]{2}{*}{ PRKAA1 } & 5 & rs461404 & 1st-primer & ACGTTGGATGGTAACATGCATTAGGCACTG & tttgGCTTCTCCTGCAATATCTTCCT \\
\hline & & & 2nd-primer & ACGTTGGATGCTAAGCTCAAAGGCAGAAAC & \\
\hline \multirow[t]{2}{*}{ PRKAG2 } & 7 & rs4078431 & 1st-primer & ACGTTGGATGACACCATAGGGCCTTTACTC & agggGTCCTGCGAAAGCAC \\
\hline & & & 2nd-primer & ACGTTGGATGAGAGATGATCCTTTGGGTGG & \\
\hline \multirow[t]{2}{*}{ PRKAR1B } & 7 & rs4724904 & 1st-primer & ACGTTGGATGTGGTTTCCAGGTATCTGGAC & tccaaCCCACCCGGGCCCACGAGC \\
\hline & & & 2 nd-primer & ACGTTGGATGCACCCTCCTCCCTGCCTGA & \\
\hline \multirow[t]{2}{*}{$G C K$} & 7 & rs12702070 & 1st-primer & ACGTTGGATGATCTGAACAGGTGGCAAAGG & CACTACAGTGACCGC \\
\hline & & & 2nd-primer & ACGTTGGATGACCATGGTGACCACTCAATC & \\
\hline \multirow[t]{2}{*}{$P R K A C G$} & 9 & rs4745515 & 1st-primer & ACGTTGGATGTTCTAAGCTCCCTTTTCATC & ATCCCCAAGTATTTGGAAA \\
\hline & & & 2nd-primer & ACGTTGGATGCTTGAAAAATCCCCAAGTAT & \\
\hline \multirow[t]{2}{*}{$F B P 1$} & 9 & rs7031924 & 1st-primer & ACGTTGGATGTTTTGAAGGTAGAGCTGATG & TCTCACATATCTCATCTGTCAA \\
\hline & & & 2nd-primer & ACGTTGGATGAGTTTCTCAGGCCTCAAACC & \\
\hline \multirow[t]{2}{*}{ HRAS } & 11 & rs7939028 & 1st-primer & ACGTTGGATGAGCCGAGCTCTGACTGCGA & ggGGTCGCTGCTGACCCG \\
\hline & & & 2nd-primer & ACGTTGGATGTCCTGCGCCGGTCGCTGCT & \\
\hline \multirow[t]{2}{*}{ PRKAB1 } & 12 & rs6490265 & 1st-primer & ACGTTGGATGTTCCGGCGACTCCGTAAGT & AGCCTCGCGCGTTTAGC \\
\hline & & & 2 nd-primer & ACGTTGGATGTGGAATCGAGATAGCCTCGC & \\
\hline \multirow[t]{2}{*}{ PRKAG1 } & 12 & rs2293446 & 1st-primer & ACGTTGGATGCATGACCCTCGCCGTCAGC & tcAACCGCCCTCCCCCGGGTCCTC \\
\hline & & & 2nd-primer & ACGTTGGATGAGGCAAGGAACCCACCCTTC & \\
\hline \multirow[t]{2}{*}{ KRAS } & 12 & rs7311692 & 1st-primer & ACGTTGGATGATAGTAAATGGATGCGCGCC & cctccGACCGGTCTCCACAGAGAAG \\
\hline & & & 2nd-primer & ACGTTGGATGCCGCTGACCGGTCTCCACA & \\
\hline \multirow[t]{2}{*}{ PCK2 } & 14 & rs4982856 & 1 st-primer & ACGTTGGATGCAGCCGCACATGATGTAACT & AGCTTGTTTGCCACC \\
\hline & & & 2nd-primer & ACGTTGGATGCCCTCGTTCCTAGCTTGTTT & \\
\hline \multirow[t]{2}{*}{ SOS 2} & 14 & rs1955926 & 1st-primer & ACGTTGGATGCCCAGACTCCAGCTATAAAC & tgCATGGTCTAGTAGTTTGACA \\
\hline & & & 2nd-primer & ACGTTGGATGCTCCTATGAGTAAAGAGAAC & \\
\hline SHC4 & 15 & rs12900666 & 1st-primer & ACGTTGGATGACTACAGATCATTAGCCGGG & сссаTTCTGCTCTGCCСТCCC \\
\hline & & & 2nd-primer & ACGTTGGATGTTTTAGCTCTTCTGCTCTGC & \\
\hline$P D P K 1$ & 16 & rs76318740 & 1st-primer & ACGTTGGATGCCAAGGGTCAAGGGTCACAA & CTGCСССТСТСТССС \\
\hline & & & 2nd-primer & ACGTTGGATGTTTGACCTTCAGCTGCCCCT & \\
\hline FASN & 17 & rs7222326 & 1st-primer & ACGTTGGATGCCTCCCTTTTCTGACCGCT & GTGCCTGGCCCCGCA \\
\hline & & & 2nd-primer & ACGTTGGATGAGACACCTGTGGCCTCACAA & \\
\hline$A C A C A$ & 17 & rs11868124 & 1st-primer & ACGTTGGATGAGAACGCGCTGTTGCACTC & tGGAGTTTCACACTTGTTGCT \\
\hline & & & 2nd-primer & ACGTTGGATGTGTGCATCCTTTACTGGACG & \\
\hline FLOT2 & 17 & rs4795473 & 1st-primer & ACGTTGGATGGTAAATACACACCACTGTGC & CAGGAAGAAAGAAAGGAGT \\
\hline & & & 2 nd-primer & ACGTTGGATGAGGAACAGCTCCGAGTTCAC & \\
\hline INSR & 19 & rs1864009 & 1st-primer & ACGTTGGATGCAGCGACACTCGTCCCAGA & CTGGGCCGGGGCGCAC \\
\hline & & & 2 nd-primer & ACGTTGGATGTGGCTTGGGTCTCGCCCCT & \\
\hline PIK3R2 & 19 & rs3736328 & 1st-primer & ACGTTGGATGTATTTCCAGAAACGCTCCGC & GGCCGAACACTCTCTAC \\
\hline & & & 2nd-primer & ACGTTGGATGTTTCGGAAATGGCCGAACAC & \\
\hline$C B L C$ & 19 & rs2965143 & 1st-primer & ACGTTGGATGTGTAGTCCTAGTTACTAGGG & ttccTTGCAGCCTTGACCTC \\
\hline & & & 2nd-primer & ACGTTGGATGTGGTTGTTGGATCACCACTC & \\
\hline$M K N K 2$ & 19 & rs3810412 & 1st-primer & ACGTTGGATGGAAGAAGAAAGTAGGTGGCG & GCACGCTTGGAGTCCCA \\
\hline & & & 2nd-primer & ACGTTGGATGTGCACCACCAGCACGCTTG & \\
\hline SHC2 & 19 & rs73916989 & 1st-primer & ACGTTGGATGTGAGGAGAGAGCAGCCCCA & aaGCAGGGCGCAGAGAG \\
\hline & & & 2nd-primer & ACGTTGGATGTTGGGAGGGTGCGACACTG & \\
\hline TRIP10 & 19 & rs340141 & 1st-primer & ACGTTGGATGCAAGAAGTCCTTCCTCAACC & ttgcCCTAGACGCCTGGAGC \\
\hline & & & 2nd-primer & ACGTTGGATGTTAGTGGGACGAACCTAGAC & \\
\hline$P R K X$ & $\operatorname{chrX}$ & rs1003351 & 1st-primer & ACGTTGGATGTGCACCCAAGCCCGGAAGAT & ccctACTTCTGCTGAGCTAC \\
\hline & & & 2nd-primer & ACGTTGGATGGTTTTGAACTTCACTTCTGC & \\
\hline
\end{tabular}

Table 2. Association of 29 variants with type 2 diabetes and prediabetes in the whole sample set 


\begin{tabular}{|c|c|c|c|c|c|c|c|c|c|c|c|c|c|}
\hline \multirow{4}{*}{$P I K 3 C D(r s 7518602)$} & \multirow{2}{*}{\multicolumn{3}{|c|}{$\begin{array}{l}\text { Genotype } \\
\text { (counts) }\end{array}$}} & \multirow[t]{2}{*}{$\mathrm{X}^{2}$} & \multirow[t]{2}{*}{$\mathrm{P}$} & \multirow[t]{2}{*}{ HWE } & \multirow{2}{*}{\multicolumn{2}{|c|}{$\begin{array}{c}\text { Allele } \\
\text { (counts) }\end{array}$}} & \multirow{3}{*}{$\begin{array}{c}\mathrm{P} \\
\text { (Adjusted) }\end{array}$} & \multirow{2}{*}{$\begin{array}{l}\text { OR(95\%CI) } \\
\text { (Adjusted) }\end{array}$} & \multicolumn{2}{|c|}{ Dominant model } & \multirow{2}{*}{$\begin{array}{r}\mathrm{R} \epsilon \\
\mathrm{P} \\
\text { (Adjus } \\
\end{array}$} \\
\hline & & & & & & & & & & & & & \\
\hline & & & & & & & & & & & & & \\
\hline & CC & CT & $\mathrm{TT}$ & & & & $\mathrm{C}$ & $\mathrm{T}$ & & & & & \\
\hline $\mathrm{T} 2 \mathrm{D}$ & 557 & 109 & 5 & 1.832 & 0.4 & 1.000 & 1223 & 119 & $0.229^{\mathrm{a}}$ & $\begin{array}{c}0.826(0.605- \\
1.128)^{\mathrm{a}}\end{array}$ & $0.302^{\mathrm{a}}$ & $\begin{array}{c}1.195(0.852- \\
1.677)^{\mathrm{a}}\end{array}$ & 0.29 \\
\hline Prediabetes & 197 & 47 & 2 & 0.329 & 0.848 & 1.000 & 441 & 51 & $0.584^{b}$ & $\begin{array}{c}1.114(0.757- \\
1.639)^{b}\end{array}$ & $0.48^{b}$ & $\begin{array}{c}0.858(0.561- \\
1.312)^{b}\end{array}$ & 0.70 । \\
\hline Control & 455 & 104 & 7 & & & 0.653 & 1014 & 118 & & & & & \\
\hline$M K N K 1(\mathrm{rs} 3753359)$ & GG & CG & $\mathrm{CC}$ & & & & G & $\mathrm{C}$ & & & & & \\
\hline $\mathrm{T} 2 \mathrm{D}$ & 409 & 260 & 34 & 2.824 & 0.244 & 0.401 & 1078 & 328 & $0.864^{\mathrm{a}}$ & $\begin{array}{c}1.019(0.820- \\
1.267)^{\mathrm{a}}\end{array}$ & $0.826^{a}$ & $\begin{array}{c}0.971(0.744- \\
1.266)^{\mathrm{a}}\end{array}$ & 0.351 \\
\hline Prediabetes & 153 & 87 & 12 & 1.139 & 0.566 & 1.000 & 393 & 111 & $0.84^{b}$ & $\begin{array}{c}1.029(0.778- \\
1.362)^{b}\end{array}$ & $0.985^{b}$ & $\begin{array}{c}0.997(0.706- \\
1.408)^{b}\end{array}$ & $0.57 i$ \\
\hline Control & 328 & 187 & 37 & & & 0.157 & 843 & 261 & & & & & \\
\hline$P P P 1 C B(\mathrm{rs} 7598876)$ & $\mathrm{CC}$ & CT & $\mathrm{TT}$ & & & & $\mathrm{C}$ & $\mathrm{T}$ & & & & & \\
\hline $\mathrm{T} 2 \mathrm{D}$ & 379 & 269 & 55 & 4.558 & 0.102 & 0.448 & 1027 & 379 & $0.402^{\mathrm{a}}$ & $\begin{array}{c}0.914(0.740- \\
1.128)^{\mathrm{a}}\end{array}$ & $0.142^{\mathrm{a}}$ & $\begin{array}{c}1.218(0.936- \\
1.586)^{\mathrm{a}}\end{array}$ & $0.40^{\prime}$ \\
\hline Prediabetes & 143 & 95 & 15 & 1.37 & 0.504 & 1.000 & 381 & 125 & $0.232^{b}$ & $\begin{array}{c}0.843(0.636- \\
1.116)^{b}\end{array}$ & $0.21^{b}$ & $\begin{array}{c}1.242(0.885- \\
1.743)^{b}\end{array}$ & $0.66 !$ \\
\hline Control & 294 & 231 & 28 & & & 0.046 & 819 & 287 & & & & & \\
\hline$P R K C I(\mathrm{rs} 481781)$ & CC & $\mathrm{CT}$ & $\mathrm{TT}$ & & & & $\mathrm{C}$ & $\mathrm{T}$ & & & & & \\
\hline $\mathrm{T} 2 \mathrm{D}$ & 454 & 213 & 36 & 1.174 & 0.556 & 0.101 & 1121 & 285 & $0.116^{\mathrm{a}}$ & $\begin{array}{c}0.837(0.670- \\
1.045)^{\mathrm{a}}\end{array}$ & $0.132^{\mathrm{a}}$ & $\begin{array}{c}1.236(0.938- \\
1.629)^{\mathrm{a}}\end{array}$ & 0.35 \\
\hline Prediabetes & 159 & 83 & 11 & 0.893 & 0.64 & 1.000 & 401 & 105 & $0.515^{b}$ & $\begin{array}{c}0.911(0.687- \\
1.207)^{b}\end{array}$ & $0.738^{b}$ & $\begin{array}{c}1.061(0.750- \\
1.500)^{b}\end{array}$ & $0.32 !$ \\
\hline Control & 341 & 178 & 33 & & & 0.138 & 860 & 244 & & & & & \\
\hline$M A P K 9(\mathrm{rs} 1363513)$ & $\mathrm{TT}$ & TG & GG & & & & $\mathrm{T}$ & G & & & & & \\
\hline $\mathrm{T} 2 \mathrm{D}$ & 459 & 193 & 24 & 3.575 & 0.167 & 0.515 & 1111 & 241 & $0.05^{\mathrm{a}}$ & $\begin{array}{c}0.780(0.608- \\
1.000)^{\mathrm{a}}\end{array}$ & $0.087^{\mathrm{a}}$ & $\begin{array}{c}0.782(0.90- \\
1.036)^{\mathrm{a}}\end{array}$ & 0.14 \\
\hline Prediabetes & 169 & 70 & 7 & 0.921 & 0.631 & 1.000 & 408 & 84 & $0.251^{b}$ & $\begin{array}{c}0.827(0.598- \\
1.144)^{b}\end{array}$ & $0.318^{b}$ & $\begin{array}{c}0.831(0.577- \\
1.196)^{b}\end{array}$ & $0.37 i$ \\
\hline Control & 402 & 152 & 11 & & & 0.521 & 956 & 174 & & & & & \\
\hline$P R K A A 1(\mathrm{rs} 461404$ ) & $\mathrm{TT}$ & $\mathrm{CT}$ & $\mathrm{CC}$ & & & & $\mathrm{T}$ & $\mathrm{C}$ & & & & & \\
\hline $\mathrm{T} 2 \mathrm{D}$ & 393 & 245 & 38 & 0.089 & 0.957 & 1.000 & 1031 & 321 & $0.616^{\mathrm{a}}$ & $\begin{array}{c}0.946(0.763- \\
1.173)^{\mathrm{a}}\end{array}$ & $0.432^{\mathrm{a}}$ & $\begin{array}{c}1.249(0.717- \\
2.176)^{\mathrm{a}}\end{array}$ & $0.80 !$ \\
\hline Prediabetes & 145 & 83 & 17 & 0.52 & 0.771 & 0.292 & 373 & 117 & $0.326^{b}$ & $\begin{array}{c}0.872(0.663- \\
1.146)^{b}\end{array}$ & $0.31^{b}$ & $\begin{array}{c}1.414(0.724- \\
2.762)^{b}\end{array}$ & 0.47 \\
\hline Control & 327 & 204 & 34 & & & 0.819 & 858 & 272 & & & & & \\
\hline PRKAG2(rs4078431) & GG & AG & AA & & & & G & A & & & & & \\
\hline $\mathrm{T} 2 \mathrm{D}$ & 168 & 362 & 173 & 0.94 & 0.625 & 0.449 & 698 & 708 & $0.238^{\mathrm{a}}$ & $\begin{array}{c}0.894(0.743- \\
1.077)^{\mathrm{a}}\end{array}$ & $0.414^{\mathrm{a}}$ & $\begin{array}{c}0.882(0.653- \\
1.191)^{\mathrm{a}}\end{array}$ & 0.68 \\
\hline Prediabetes & 58 & 122 & 73 & 2.375 & 0.305 & 0.615 & 238 & 268 & $0.033^{b}$ & $\begin{array}{c}0.770(0.606- \\
0.979)^{b}\end{array}$ & $0.185^{b}$ & $\begin{array}{c}0.766(0.516- \\
1.136)^{b}\end{array}$ & 0.03 \\
\hline Control & 145 & 274 & 133 & & & 0.866 & 564 & 540 & & & & & \\
\hline$P R K A R 1 B(\mathrm{rs} 4724904)$ & $\mathrm{CC}$ & CG & GG & & & & $\mathrm{C}$ & G & & & & & \\
\hline $\mathrm{T} 2 \mathrm{D}$ & 288 & 320 & 95 & 0.302 & 0.86 & 0.680 & 896 & 510 & $0.785^{\mathrm{a}}$ & $\begin{array}{c}1.027(0.8437- \\
1.246)^{\mathrm{a}}\end{array}$ & $0.79^{a}$ & $\begin{array}{c}0.964(0.739- \\
1.258)^{\mathrm{a}}\end{array}$ & $0.87:$ \\
\hline Prediabetes & 96 & 122 & 35 & 1.136 & 0.567 & 0.788 & 314 & 192 & $0.364^{b}$ & $\begin{array}{c}1.121(0.876- \\
1.433)^{b}\end{array}$ & $0.237^{\mathrm{b}}$ & $\begin{array}{c}0.812(0.575- \\
1.147)^{b}\end{array}$ & 0.91 \\
\hline Control & 231 & 252 & 69 & & & 1.000 & 714 & 390 & & & & & \\
\hline$G C K(\operatorname{rs} 12702070)$ & TT & CT & $\mathrm{CC}$ & & & & $\mathrm{T}$ & $\mathrm{C}$ & & & & & \\
\hline $\mathrm{T} 2 \mathrm{D}$ & 543 & 126 & 6 & 3.233 & 0.186 & 0.834 & 1212 & 138 & $0.729^{\mathrm{a}}$ & $\begin{array}{c}0.941(0.669- \\
1.325)^{\mathrm{a}}\end{array}$ & $0.926^{a}$ & $\begin{array}{c}1.018(0.701- \\
1.478)^{\mathrm{a}}\end{array}$ & 0.28 \\
\hline Prediabetes & 197 & 44 & 4 & 2.611 & 0.261 & 0.328 & 438 & 52 & $0.039 \mathrm{~b}$ & $\begin{array}{c}1.526(1.021- \\
2.279)^{b}\end{array}$ & $0.057^{b}$ & $\begin{array}{c}0.654(0.423- \\
1.013)^{b}\end{array}$ & 0.19 \\
\hline Control & 477 & 86 & 4 & & & & & & & & & & \\
\hline$P R K A C G(\mathrm{rs} 4745515$ ) & AA & AG & GG & & & & $\mathrm{A}$ & G & & & & & \\
\hline $\mathrm{T} 2 \mathrm{D}$ & 322 & 287 & 67 & 1.901 & 0.387 & 0.788 & 931 & 421 & $0.559^{a}$ & $\begin{array}{c}1.059(0.873- \\
1.286)^{\mathrm{a}}\end{array}$ & $0.324^{\mathrm{a}}$ & $\begin{array}{c}0.877(0.675- \\
1.139)^{\mathrm{a}}\end{array}$ & 0.75 \\
\hline Prediabetes & 115 & 106 & 24 & 1.125 & 0.57 & 1.000 & 336 & 154 & $0.38^{b}$ & $\begin{array}{c}1.118(0.872- \\
1.434)^{b}\end{array}$ & $0.127^{b}$ & $\begin{array}{c}0.766(0.544- \\
1.079)^{b}\end{array}$ & 0.60 : \\
\hline Control & 261 & 235 & 70 & & & 0.128 & 757 & 375 & & & & & \\
\hline$F B P 1($ rs7031924) & GG & AG & AA & & & & G & A & & & & & \\
\hline $\mathrm{T} 2 \mathrm{D}$ & 527 & 166 & 10 & 0.173 & 0.917 & 0.514 & 1220 & 186 & $0.511^{\mathrm{a}}$ & $\begin{array}{c}1.097(0.832- \\
1.446)^{\mathrm{a}}\end{array}$ & $0.608^{a}$ & $\begin{array}{c}0.923(0.681- \\
1.252)^{\mathrm{a}}\end{array}$ & 0.47 \\
\hline Prediabetes & 178 & 74 & 1 & 4.931 & 0.085 & 0.023 & 430 & 76 & $0.57^{\mathrm{b}}$ & $\begin{array}{c}1.109(0.776- \\
1.587)^{b}\end{array}$ & $0.306^{\mathrm{b}}$ & $\begin{array}{c}0.821(0.563- \\
1.197)^{b}\end{array}$ & $0.99 !$ \\
\hline Control & 419 & 126 & 7 & & & 0.568 & 964 & 140 & & & & & \\
\hline$H R A S($ rs7939028) & $\mathrm{CC}$ & CG & GG & & & & $\mathrm{C}$ & G & & & & & \\
\hline $\mathrm{T} 2 \mathrm{D}$ & 457 & 220 & 26 & 2.776 & 0.25 & 1.000 & 1134 & 272 & $0.784^{\mathrm{a}}$ & $\begin{array}{c}1.034(0.812- \\
1.317)^{\mathrm{a}}\end{array}$ & $0.843^{\mathrm{a}}$ & $\begin{array}{c}1.028(0.781- \\
1.353)^{\mathrm{a}}\end{array}$ & $0.15 !$ \\
\hline Prediabetes & 178 & 68 & 7 & 3.413 & 0.182 & 0.818 & 424 & 82 & $0.429 \mathrm{~b}$ & $\begin{array}{c}0.878(0.653- \\
1.213)^{b}\end{array}$ & $0.213^{b}$ & $\begin{array}{c}1.245(0.870- \\
1.782)^{b}\end{array}$ & $0.31:$ \\
\hline Control & 357 & 184 & 12 & & & 0.037 & 898 & 208 & & & & & \\
\hline$P R K A B 1(\mathrm{rs} 6490265)$ & $\mathrm{CC}$ & $\mathrm{CT}$ & $\mathrm{TT}$ & & & & $\mathrm{C}$ & $\mathrm{T}$ & & & & & \\
\hline
\end{tabular}




\begin{tabular}{|c|c|c|c|c|c|c|c|c|c|c|c|c|c|}
\hline $\mathrm{T} 2 \mathrm{D}$ & 189 & 350 & 163 & 1.676 & 0.433 & 1.000 & 728 & 676 & $0.011^{\mathrm{a}}$ & $\begin{array}{c}1.270(1.055- \\
1.528)^{\mathrm{a}}\end{array}$ & $0.034^{\mathrm{a}}$ & $\begin{array}{c}0.731(0.546- \\
0.977)^{\mathrm{a}}\end{array}$ & $0.04:$ \\
\hline Prediabetes & 91 & 118 & 44 & 3.377 & 0.185 & 0.606 & 300 & 206 & $0.292^{b}$ & $\begin{array}{c}0.881(0.696- \\
1.115)^{\mathrm{b}}\end{array}$ & $0.37^{\mathrm{b}}$ & $\begin{array}{c}1.176(0.825- \\
1.677)^{b}\end{array}$ & $0.40 !$ \\
\hline Control & 165 & 271 & 116 & & & 0.795 & 601 & 503 & & & & & \\
\hline PRKAG1(rs2293446) & GG & GA & AA & & & & G & $\mathrm{A}$ & & & & & \\
\hline $\mathrm{T} 2 \mathrm{D}$ & 230 & 320 & 126 & 0.232 & 0.891 & 0.434 & 780 & 572 & $0.792^{\mathrm{a}}$ & $\begin{array}{c}1.025(0.852- \\
1.233)^{\mathrm{a}}\end{array}$ & $0.804^{\mathrm{a}}$ & $\begin{array}{c}0.965(0.732- \\
1.273)^{\mathrm{a}}\end{array}$ & $0.43 i$ \\
\hline Prediabetes & 82 & 113 & 50 & 0.424 & 0.809 & 0.362 & 277 & 213 & $0.716^{b}$ & $\begin{array}{c}0.958(0.758- \\
1.210)^{b}\end{array}$ & $0.715^{b}$ & $\begin{array}{c}0.936(0.655- \\
1.336)^{b}\end{array}$ & 0.82 \\
\hline Control & 184 & 272 & 106 & & & 0.797 & 640 & 484 & & & & & \\
\hline$K R A S(\mathrm{rs} 7311692)$ & $\mathrm{CC}$ & CG & GG & & & & $\mathrm{C}$ & G & & & & & \\
\hline $\mathrm{T} 2 \mathrm{D}$ & 576 & 121 & 6 & 2.971 & 0.226 & 1.000 & 1273 & 133 & $0.552^{\mathrm{a}}$ & $\begin{array}{c}0.911(0.671- \\
1.238)^{\mathrm{a}}\end{array}$ & $0.805^{a}$ & $\begin{array}{c}1.043(0.746- \\
1.458)^{\mathrm{a}}\end{array}$ & 0.14 \\
\hline Prediabetes & 220 & 32 & 1 & 0.923 & 0.63 & 1.000 & 472 & 34 & $0.009^{b}$ & $\begin{array}{c}0.562(0.363- \\
0.868)^{b}\end{array}$ & $0.023^{b}$ & $\begin{array}{c}1.736(1.078- \\
2.797)^{b}\end{array}$ & 0.05 \\
\hline Control & 450 & 93 & 9 & & & 0.152 & 993 & 111 & & & & & \\
\hline PCK2(rs4982856) & $\mathrm{CC}$ & CT & TT & & & & $\mathrm{C}$ & $\mathrm{T}$ & & & & & \\
\hline $\mathrm{T} 2 \mathrm{D}$ & 174 & 368 & 160 & 2.835 & 0.242 & 0.223 & 716 & 688 & $0.758^{a}$ & $\begin{array}{c}0.971(0.807- \\
1.169)^{\mathrm{a}}\end{array}$ & $0.668^{a}$ & $\begin{array}{c}0.937(0.698- \\
1.260)^{\mathrm{a}}\end{array}$ & $0.33^{\prime}$ \\
\hline Prediabetes & 66 & 137 & 50 & 2.553 & 0.279 & 0.207 & 269 & 237 & $0.6^{\mathrm{b}}$ & $\begin{array}{c}0.938(0.740- \\
1.190)^{b}\end{array}$ & $0.632^{b}$ & $\begin{array}{c}0.911(0.623- \\
1.333)^{b}\end{array}$ & $0.16 i$ \\
\hline Control & 158 & 266 & 128 & & & 0.446 & 582 & 522 & & & & & \\
\hline SOS2( rs1955926) & $\mathrm{CC}$ & $\mathrm{CA}$ & AA & & & & $\mathrm{C}$ & A & & & & & \\
\hline $\mathrm{T} 2 \mathrm{D}$ & 294 & 295 & 87 & 0.901 & 0.637 & 0.357 & 883 & 469 & $0.464^{\mathrm{a}}$ & $\begin{array}{c}1.074(0.887- \\
1.300)^{\mathrm{a}}\end{array}$ & $0.379^{a}$ & $\begin{array}{c}1.125(0.865- \\
1.464)^{\mathrm{a}}\end{array}$ & $0.84 i$ \\
\hline Prediabetes & 106 & 111 & 29 & 0.282 & 0.868 & 1.000 & 323 & 169 & $0.558^{b}$ & $\begin{array}{c}1.078(0.839- \\
1.383)^{\mathrm{b}}\end{array}$ & $0.559^{b}$ & $\begin{array}{c}1.107(0.787- \\
1.556)^{b}\end{array}$ & 0.741 \\
\hline Control & 233 & 262 & 71 & & & 0.927 & 728 & 404 & & & & & \\
\hline SHC4( rs12900666) & $\mathrm{CC}$ & $\mathrm{CA}$ & AA & & & & $\mathrm{C}$ & A & & & & & \\
\hline T2D & 356 & 267 & 52 & 2.971 & 0.226 & 0.848 & 979 & 371 & $0.604^{\mathrm{a}}$ & $\begin{array}{c}0.945(0.763- \\
1.170)^{\mathrm{a}}\end{array}$ & $0.824^{a}$ & $\begin{array}{c}0.971(0.747- \\
1.261)^{\mathrm{a}}\end{array}$ & $0.39:$ \\
\hline Prediabetes & 130 & 98 & 17 & 0.923 & 0.63 & 0.872 & 358 & 132 & $0.283^{b}$ & $\begin{array}{c}0.859(0.650- \\
1.134)^{b}\end{array}$ & $0.426^{b}$ & $\begin{array}{c}0.871(0.620- \\
1.223)^{\mathrm{b}}\end{array}$ & 0.28 \\
\hline Control & 311 & 222 & 30 & & & 0.267 & 844 & 282 & & & & & \\
\hline$P D P K 1(\mathrm{rs} 76318740)$ & $\mathrm{CC}$ & CT & TT & & & & $\mathrm{C}$ & $\mathrm{T}$ & & & & & \\
\hline $\mathrm{T} 2 \mathrm{D}$ & 512 & 159 & 5 & 0.2801 & 0.246 & 0.052 & 1183 & 169 & $0.902^{\mathrm{a}}$ & $\begin{array}{c}0.983(0.744- \\
1.298)^{\mathrm{a}}\end{array}$ & $0.666^{\mathrm{a}}$ & $\begin{array}{c}0.935(0.690- \\
1.267)^{\mathrm{a}}\end{array}$ & 0.661 \\
\hline Prediabetes & 180 & 60 & 6 & 0.764 & 0.683 & 0.618 & 420 & 72 & $0.354^{b}$ & $\begin{array}{c}1.175(0.836- \\
1.651)^{b}\end{array}$ & $0.581^{b}$ & $\begin{array}{c}0.881(0.599- \\
1.295)^{b}\end{array}$ & 0.451 \\
\hline Control & 428 & 128 & 10 & & & 0.854 & & & & & & & \\
\hline FASN(rs7222326) & TT & CT & $\mathrm{CC}$ & & & & $\mathrm{T}$ & $\mathrm{C}$ & & & & & \\
\hline $\mathrm{T} 2 \mathrm{D}$ & 521 & 142 & 12 & 3.035 & 0.219 & 0.478 & 1184 & 166 & $0.087^{\mathrm{a}}$ & $\begin{array}{c}1.286(0.964- \\
1.717)^{\mathrm{a}}\end{array}$ & $0.157^{\mathrm{a}}$ & $\begin{array}{c}0.797(0.581- \\
1.091)^{\mathrm{a}}\end{array}$ & 0.11 \\
\hline Prediabetes & 197 & 47 & 1 & 0.342 & 0.945 & 0.483 & 441 & 49 & $0.261^{\mathrm{b}}$ & $\begin{array}{c}0.791(0.526- \\
1.191)^{\mathrm{b}}\end{array}$ & $0.329^{b}$ & $\begin{array}{c}1.237(0.807- \\
1.896)^{b}\end{array}$ & $0.32:$ \\
\hline Control & 447 & 113 & 4 & & & 0.379 & 1007 & 121 & & & & & \\
\hline$A C A C A(\mathrm{rs} 11868124)$ & $\mathrm{CC}$ & CT & TT & & & & $\mathrm{C}$ & $\mathrm{T}$ & & & & & \\
\hline $\mathrm{T} 2 \mathrm{D}$ & 466 & 185 & 25 & 0.354 & 0.838 & 0.230 & 1117 & 235 & $0.95^{\mathrm{a}}$ & $\begin{array}{c}1.008(0.796- \\
1.276)^{\mathrm{a}}\end{array}$ & $0.811^{\mathrm{a}}$ & $\begin{array}{c}0.966(0.731- \\
1.278)^{\mathrm{a}}\end{array}$ & 0.68 \\
\hline Prediabetes & 166 & 71 & 9 & 0.084 & 0.959 & 0.666 & 403 & 89 & $0.29^{b}$ & $\begin{array}{c}1.174(0.872- \\
1.580)^{b}\end{array}$ & $0.286^{b}$ & $\begin{array}{c}0.824(0.577- \\
1.176)^{b}\end{array}$ & $0.64:$ \\
\hline Control & 388 & 158 & 21 & & & 0.315 & 934 & 200 & & & & & \\
\hline FLOT2(rs4795473) & $\mathrm{TT}$ & CT & $\mathrm{CC}$ & & & & $\mathrm{T}$ & $\mathrm{C}$ & & & & & \\
\hline $\mathrm{T} 2 \mathrm{D}$ & 352 & 301 & 50 & 4.911 & 0.086 & 0.193 & 1005 & 401 & $0.272^{\mathrm{a}}$ & $\begin{array}{c}1.121(0.914- \\
1.374)^{\mathrm{a}}\end{array}$ & $0.931^{\mathrm{a}}$ & $\begin{array}{c}1.012(0.779- \\
1.313)^{\mathrm{a}}\end{array}$ & $0.01 i$ \\
\hline Prediabetes & 121 & 116 & 16 & 0.749 & 0.687 & 0.099 & 358 & 148 & $0.36^{b}$ & $\begin{array}{c}1.129(0.871- \\
1.464)^{b}\end{array}$ & $0.998^{b}$ & $\begin{array}{c}1.000(0.716- \\
1.398)^{\mathrm{b}}\end{array}$ & 0.04 \\
\hline Control & 281 & 215 & 57 & & & 0.103 & 777 & 329 & & & & & \\
\hline$I N S R(\mathrm{rs} 1864009)$ & GG & GT & TT & & & & G & $\mathrm{T}$ & & & & & \\
\hline $\mathrm{T} 2 \mathrm{D}$ & 166 & 351 & 159 & 2.070 & 0.355 & 0.354 & 683 & 669 & $0.305^{a}$ & $\begin{array}{c}0.907(0.753- \\
1.093)^{\mathrm{a}}\end{array}$ & $0.523^{a}$ & $\begin{array}{c}1.105(0.813- \\
1.504)^{\mathrm{a}}\end{array}$ & $0.30 !$ \\
\hline Prediabetes & 45 & 125 & 76 & 2.388 & 0.303 & 0.695 & 215 & 277 & $0.192^{b}$ & $\begin{array}{c}1.173(0.923- \\
1.491)^{\mathrm{b}}\end{array}$ & $0.231^{b}$ & $\begin{array}{c}0.772(0.505- \\
1.179)^{b}\end{array}$ & 0.341 \\
\hline Control & 127 & 286 & 152 & & & 0.799 & 540 & 590 & & & & & \\
\hline PIK3R2(rs3736328) & $\mathrm{CC}$ & CG & GG & & & & $\mathrm{C}$ & G & & & & & \\
\hline $\mathrm{T} 2 \mathrm{D}$ & 376 & 252 & 48 & 3.196 & 0.202 & 0.547 & 1004 & 348 & $0.631^{\mathrm{a}}$ & $\begin{array}{c}1.053(0.853- \\
1.300)^{\mathrm{a}}\end{array}$ & $0.799^{\mathrm{a}}$ & $\begin{array}{c}1.034(0.799- \\
1.338)^{\mathrm{a}}\end{array}$ & $0.07^{\prime}$ \\
\hline Prediabetes & 144 & 87 & 15 & 1.6 & 0.449 & 0.723 & 375 & 117 & $0.577^{\mathrm{b}}$ & $\begin{array}{c}0.922(0.695- \\
1.255)^{b}\end{array}$ & $0.285^{b}$ & $\begin{array}{c}1.204(0.857- \\
1.690)^{\mathrm{b}}\end{array}$ & 0.37 \\
\hline Control & 316 & 224 & 27 & & & 0.138 & 856 & 278 & & & & & \\
\hline$C B L C(\mathrm{rs} 2965143)$ & $\mathrm{AA}$ & AG & GG & & & & A & G & & & & & \\
\hline $\mathrm{T} 2 \mathrm{D}$ & 216 & 349 & 111 & 3.886 & 0.143 & 0.154 & 781 & 571 & $0.239^{a}$ & $\begin{array}{c}1.122(0.926- \\
1.360)^{\mathrm{a}}\end{array}$ & $0.342^{\mathrm{a}}$ & $\begin{array}{c}0.875(0.663- \\
1.153)^{\mathrm{a}}\end{array}$ & 0.33 \\
\hline Prediabetes & 110 & 99 & 37 & 6.388 & 0.041 & 0.069 & 319 & 173 & $0.04^{b}$ & $\begin{array}{c}0.772(0.604- \\
0.988)^{b}\end{array}$ & $0.008^{b}$ & $\begin{array}{c}1.591(1.128- \\
2.242)^{b}\end{array}$ & $0.71^{\prime}$ \\
\hline Control & 208 & 282 & 77 & & & 0.247 & 698 & 436 & & & & & \\
\hline$M K N K 2(\mathrm{rs} 3810412)$ & $\mathrm{CC}$ & CT & $\mathrm{TT}$ & & & & $\mathrm{C}$ & $\mathrm{T}$ & & & & & \\
\hline
\end{tabular}




\begin{tabular}{|c|c|c|c|c|c|c|c|c|c|c|c|c|c|}
\hline $\mathrm{T} 2 \mathrm{D}$ & 557 & 138 & 8 & 1.64 & 0.44 & 1.000 & 1252 & 154 & $0.204^{\mathrm{a}}$ & $\begin{array}{c}1.215(0.899- \\
1.642)^{\mathrm{a}}\end{array}$ & $0.231^{\mathrm{a}}$ & $\begin{array}{c}0.818(0.589- \\
1.136)^{\mathrm{a}}\end{array}$ & 0.48 \\
\hline Prediabetes & 213 & 38 & 2 & - & - & 0.683 & 464 & 42 & $0.727^{b}$ & $\begin{array}{c}0.929(0.612- \\
1.408)^{b}\end{array}$ & $0.683^{b}$ & $\begin{array}{c}1.098(0.700- \\
1.725)^{b}\end{array}$ & 0.91 \\
\hline Control & 454 & 94 & 5 & 3.565 & 0.468 & 1.000 & 1002 & 104 & & & & & \\
\hline SHC2(rs73916989) & CC & CG & GG & & & & $\mathrm{C}$ & G & & & & & \\
\hline T2D & 502 & 183 & 18 & 2.482 & 0.289 & 0.773 & 1187 & 219 & $0.465^{\mathrm{a}}$ & $\begin{array}{c}0.910(0.706- \\
1.172)^{\mathrm{a}}\end{array}$ & $0.4^{\mathrm{a}}$ & $\begin{array}{c}0.131(0.849- \\
1.509)^{\mathrm{a}}\end{array}$ & 0.96 \\
\hline Prediabetes & 175 & 71 & 6 & 0.406 & 0.816 & 0.822 & 421 & 83 & $0.859^{b}$ & $\begin{array}{c}1.030(0.743- \\
1.428)^{b}\end{array}$ & $0.904^{b}$ & $\begin{array}{c}0.978(0.678- \\
1.410) \mathrm{b}\end{array}$ & 0.80 \\
\hline Control & 379 & 163 & 10 & & & 0.127 & 921 & 183 & & & & & \\
\hline TRIP1O(rs340141) & $\mathrm{CC}$ & $\mathrm{CT}$ & $\mathrm{TT}$ & & & & $\mathrm{C}$ & $\mathrm{T}$ & & & & & \\
\hline $\mathrm{T} 2 \mathrm{D}$ & 430 & 245 & 28 & 2.214 & 0.331 & 0.371 & 1105 & 301 & $0.369^{\mathrm{a}}$ & $\begin{array}{c}0.903(0.722- \\
1.129)^{\mathrm{a}}\end{array}$ & $0.572^{a}$ & $\begin{array}{c}1.080(0.826- \\
1.413)^{\mathrm{a}}\end{array}$ & 0.24 \\
\hline Prediabetes & 171 & 76 & 6 & 6.785 & 0.034 & 0.660 & 418 & 88 & $0.061^{b}$ & $\begin{array}{c}0.748(0.552- \\
1.013)^{b}\end{array}$ & $0.13^{b}$ & $\begin{array}{c}1.314(0.922- \\
1.872)^{b}\end{array}$ & 0.091 \\
\hline Control & 332 & 189 & 32 & 8.095 & & 0.469 & 853 & 253 & & & & & \\
\hline$P R K X(\mathrm{rs} 1003351)$ & AA & AG & GG & & & & A & G & & & & & \\
\hline $\mathrm{T} 2 \mathrm{D}$ & 649 & 26 & 0 & - & - & 1.000 & 1324 & 26 & $0.187^{\mathrm{a}}$ & $\begin{array}{c}1.655(0.783- \\
3.493)^{\mathrm{a}}\end{array}$ & $0.187^{\mathrm{a}}$ & $\begin{array}{c}0.604(0.286- \\
1.276)^{\mathrm{a}}\end{array}$ & - \\
\hline Prediabetes & 237 & 8 & 0 & - & - & 1.000 & 482 & 8 & $0.54^{b}$ & $\begin{array}{c}1.343(0.523- \\
3.452)^{b}\end{array}$ & $0.54^{b}$ & $\begin{array}{c}0.745(0.290- \\
1.914)^{\mathrm{b}}\end{array}$ & - \\
\hline Control & 550 & 16 & 0 & - & - & 1.000 & 1116 & 16 & & & & & \\
\hline
\end{tabular}

a: adjusted for age, BMI,SBP, DBP,TG, IDL, Cre; b: adjusted for age, BMI,SBP, DBP,TC, TG, UA, Cre.

\section{Figures}

\section{Figure 1}

29 CpG-SNPs of genes collected from insulin signaling pathway.

\section{Supplementary Files}

This is a list of supplementary files associated with this preprint. Click to download.

- SupplementalTable2.docx

- SupplementalTable1.docx 